\title{
Validity Information Retrieval for Spatio-Temporal Queries: Theoretical Performance Bounds
}

\author{
Yufei Tao ${ }^{1}$, Nikos Mamoulis ${ }^{2}$, and Dimitris Papadias ${ }^{3}$ \\ ${ }^{1}$ Department of Computer Science \\ Carnegie Mellon University, USA, 15213-3891 \\ taoyfecs.cmu.edu \\ ${ }^{2}$ Department of Computer Science and Information Systems \\ University of Hong Kong, Hong Kong \\ nikosecsis.hku.hk \\ ${ }^{3}$ Department of Computer Science \\ Hong Kong University of Science and Technology, Hong Kong \\ dimitrisecs.ust.hk
}

\begin{abstract}
The results of traditional spatial queries (i.e., range search, nearest neighbor, etc.) are usually meaningless in spatio-temporal applications, because they will be invalidated by the movements of query and/or data objects. In practice, a query result $R$ should be accompanied with validity information specifying (i) the (future) time $T$ that $R$ will expire, and (ii) the change $C$ of $R$ at time $T$ (so that $R$ can be updated incrementally). Although several algorithms have been proposed for this problem, their worst-case performance is the same as that of sequential scan. This paper presents the first theoretical study on validity queries, and develops indexes and algorithms with attractive I/O complexities. Our discussion covers numerous important variations of the problem and different query/object mobility combinations. The solutions involve a set of nontrivial reductions that reveal the problem characteristics and permit the deployment of existing structures.
\end{abstract}

\section{Problem Formulation}

Traditional spatial query processing is insufficient in spatio-temporal applications, where the data and/or query objects change their locations. Consider, for example, a moving user who asks for the nearest hotel; the result (e.g., hotel $a$ ) of a conventional nearest neighbor query is by itself meaningless because it may be invalidated as soon as the user moves. In practice, the query result $R$ should be accompanied by additional validity information: (i) the expiry time $T$ of $R$, i.e., the future time when $a$ ceases to be the nearest hotel (based on the user's moving direction and speed), and (ii) the result change $C$ at $T$ (e.g., the next nearest hotel after $a$ expires). This problem is not specific to nearest neighbor search, but actually exists in all query types [TP02].

In this paper, we discuss validity information retrieval for the most common spatial queries, namely, (orthogonal) range search (RS) and nearest neighbor (NN) queries, considering one- or two- dimensional objects with linear movements. Following the common modeling in the literature [SJLL00, GBE+00], a moving point $p$ is represented using its location $\mathbf{p}(0)$ at the system reference time 0 , and its current velocity $\mathbf{v}$ $(\mathbf{p}(0)$ and $\mathbf{v}$ are $1 \mathrm{D} / 2 \mathrm{D}$ vectors), such that its location $\mathbf{p}(t)$ at any future time $t$ can be 
computed as $\mathbf{p}(t)=\mathbf{p}(0)+\mathbf{v} \cdot t$. Similarly, we represent a moving rectangle with two moving points that decide its opposite corners, under the constraint that they have identical velocity vectors (i.e., the extent of the rectangle remains fixed at all times). Static objects are trivially captured with zero velocities. Without loss of generality, we assume (unless specifically stated) that the system reference time 0 coincides with the current time.

The result $R$ of a spatial query is invalidated when some object "influences" it in the future, namely, (i) an object currently not in $R$ starts qualifying the query predicate, or (ii) an object originally in $R$ incurs predicate violation. A natural way to define validity retrieval is through the concept of "influence time" introduced in [TP02]. Specifically, given a RS (range search) query $q$ with result $R$ (containing all the objects currently covered by $q$ ), the influence time $T_{p}$ of data point $p$ in $R$, equals the earliest time that $p$ falls out of $q$; on the other hand, for a point $p$ not in $R, T_{p}$ corresponds to the first timestamp that $p$ lies in $q$. The concept of influence time also applies to NN (nearest neighbor) search. In this case, the influence time of a data point is the time when it will come closer to $q$ than its current NN. Here the influence time should be interpreted as the time that the object will invalidate the query result $R$, only if it has not changed earlier. Now we are ready to formulate the problem of validity information retrieval.

Problem (Validity information retrieval): Given a set of points $S=\left\{p_{1}, p_{2}, \ldots, p_{N}\right\}$ and a spatial query at the current time 0 , the corresponding validity query $q$ returns (i) the expiry time $T=\min \left\{T_{p_{i}}(1 \leq i \leq N)\right\}$, and (ii) the result change (at time $T$ ) $C=\left\{p_{i} \in S\right.$ : $\left.T_{p_{i}}=T\right\}$, where $T_{p_{i}}$ is the influence time of point $p_{i}$.

This paper presents the first study on the theoretical complexity of validity queries, aiming at solutions with good worst case performance. Our discussion is based on the popular memory-disk hierarchy [ASV99, AAE00], where each I/O access transfers a page of $B$ (i.e., page size) units of information from the disk to the main memory, which contains at least $B^{2}$ pages (a reasonable assumption in practice). The query cost is measured as the number of disk pages visited. Our objective is to achieve fast query time with small space consumption.

\subsection{Previous results}

A study of validity queries appear in [TP02] which deploys branch-and-bound algorithms on $\mathrm{R}^{*}$-trees [BKSS90] (for static objects) and TPR-trees [SJLL00] (for dynamic objects). In the worst case, these algorithms perform $O(N / B)$ I/Os (i.e., the complexity of a simple sequential scan), where $N$ is the number of objects in the dataset. All the other attempts [SR01, TPS02, BJKS02] addressing variations of the problem, incur the same complexity. On the other hand, there is a significant amount of theoretical results on conventional spatial queries for static and moving objects.

For orthogonal range search on static points, Kanth and Singh [KS99] prove that the best possible query time using any structure consuming linear $O(N / B)$ space is $O\left((N / B)^{1 / 2}+K / B\right) \mathrm{I} / \mathrm{Os}$, where $K$ is the number of objects retrieved. This bound is tight and has been realized by the $O$-tree [KS99] and the cross-tree [GI99]. Applying the theory of indexability [HKP97], Arge et al. [ASV99] show that a structure achieving optimal query cost $O\left(\log _{B}(N / B)+K / B\right)$ must occupy $\Omega\left((N / B) \log _{B}(N / B) / \log _{\log }(N / B)\right)$ 
space. They propose the external range tree that achieves these bounds. A special RS is the so-called 3-sided query, where an edge of the query rectangle lies on the boundary of the data space. Arge et al. [ASV99] design the external priority search tree that answers such queries optimally (i.e., logarithmic query cost and linear space consumption). Earlier, non-optimal structures for RS and 3-sided queries can be found in [IKO87, KRVV96, RS94, SR95].

The first study on RS queries for moving objects [KGT99a] deals with only 1D data. Agarwal et al. [AAE00] present several interesting results in the 2D space following the kinetic approach [BGH97]. In particular, they show that if queries arrive in chronological order, a RS can be answered with the same time complexity as the static case (i.e., optimally) using the kinetic external range tree. They also give two timeresponsive indexing schemes (later refined in [AAV01]) where the query cost depends on the difference between the query issue time and the current time.

Fewer results exist for nearest neighbor search in secondary memory. For static data, a Voronoi diagram can be constructed in $O(N \log N)$ time [BKOS97], after which a NN query is reduced to a point-location problem (i.e., identifying the Voronoi cell that contains the query point), which can be solved optimally using linear space and logarithmic I/O overhead [ADT03]. Agarwal et al. [AAE+00] design another solution that avoids computing the Voronoi diagram, and answers a NN query in $O\left(\log _{B}(N / B)\right)$ time, but uses non-linear $(N / B) \log (N / B)$ space. Little work has been done for NN retrieval on moving objects in external memory. Kollios et al. [KGT99b] develop various schemes, but do not prove any performance bound. Finally, several solutions exist for the different but related problem of approximate nearest neighbor search [CGR95, GLM00, AAE00]. In this paper we focus on exact NN queries.

\subsection{Our results}

For static data (and moving queries), we show that a validity RS query can be answered optimally in $O\left(\log _{B}(N / B)\right)$ I/Os using $O(N / B)$ space, for a constant number of query sizes and directions. Then, we discuss the problem where the query has arbitrary size but its movement is restricted to be axis-parallel, and develop a structure that consumes $O\left((N / B) \log _{B}(N / B)\right)$ space and answers a query in $O\left(\log _{B}(N / B)\right) \mathrm{I} / \mathrm{Os}$. Based on the external range tree, we present another solution that occupies $O\left((N / B) \log _{B}(N / B) / \log _{\log }(N / B)\right)$ space and solves a query in $O\left(\log _{B}{ }^{2}(N / B) / \log \log _{B}(N / B)\right)$ I/Os. The persistent version of this structure answers a query with arbitrary size and moving direction with the same cost but higher space complexity $O\left(\left(N^{2} / B\right) \log _{B}(N / B) / \log \log _{B}(N / B)\right)$. All the above problems can also be solved using a modified partition tree with linear space and query overhead $O\left((N / B)^{1 / 2+\varepsilon}\right)$, where $\varepsilon$ is an arbitrarily small positive number. For moving data and static RS, a validity query can be answered with optimal $O\left(\log _{B}(N / B)\right)$ I/O cost, using an index with $O\left(\left(N^{2} / B\right) \log _{B}(N / B)\right)$ space. If both objects and queries move on a linear space, we propose a solution that achieves $O\left(\log _{B}(N / B)\right)$ query time and requires $O(N / B)$ space. Further, given a Voronoi diagram on static data, a validity NN query can be answered in $\log (N / B)$ time based on an optimal external memory structure for point location queries. The same complexity can also be achieved for one-dimensional dynamic queries and objects. 
The rest of the paper is organized as follows. Section 2 reviews the previous indexes fundamental to our discussion, and elaborates the problems solved together with the corresponding query time and space complexities. Section 3 presents our results for validity RS queries, while Section 4 focuses on nearest neighbor search. Section 5 concludes the paper with a set of open problems.

\section{Preliminary Structures}

The persistent (also known as multi-version) B-tree [BGO+96] is an efficient storage scheme for a set of B-trees $B_{1}, B_{2}, \ldots, B_{\beta}$. The idea is to store in $B_{i}(2 \leq i \leq \beta)$ only the "changes" from the previous version $B_{i-1}$, while sharing the index nodes that contain their common records. Particularly, in each node, there is either none or at least $\Theta(B)$ records for any $B_{i}(1 \leq i \leq \beta)$, which guarantees that querying each $B_{i}$ has the same complexity as a normal B-tree. As proven in [BGO+96], if the total number of distinct records in all B-trees (i.e., the total number of "changes" between consecutive trees) is $O(N)$, a persistent B-tree uses linear space $O(N / B)$ and can be constructed in $O\left((N / B) \log _{B}(N / B)\right) \mathrm{I} / \mathrm{Os}$ [A94]. A range-query in any B-tree $B_{i}(1 \leq i \leq \beta)$ is answered in $O\left(\log _{B}\left(N_{i} / B\right)+\log _{B}(\beta / B)+K / B\right)=O\left(\log _{B}(N / B)+K / B\right)$ I/Os, where $N_{i}$ is the number of records in $B_{i}, \beta$ the total number of B-trees, and $K$ is the number of records retrieved. Both the space and query time complexities are optimal. As shown shortly, the persistent structure constitutes a powerful tool for our problem.

The partition tree [AAE00] is a popular 2D structure for the simplex range query, which specifies a constant number of half-planes and retrieves the set of points in their intersection (i.e., the search region). Given a set $S$ with $N$ points, the idea is to construct a balanced simplicial partition $\Lambda=\left\{\Lambda_{1}, \Lambda_{2}, \ldots, \Lambda_{r}\right\}$, where $r=O(B)$ and all points in $\Lambda_{i}(1 \leq i \leq r)$ are covered by a triangle $\Delta_{i}$. The partitions have the following properties: (i) $\Lambda_{l} \cup \Lambda_{2} \cup \ldots \cup \Lambda_{r}=S$, (ii) for any $i, j$ in $[1, r], \Lambda_{i} \cap \Lambda_{j}=\varnothing$ (i.e., the first two properties guarantee that any data point belongs to a unique partition), (iii) each $\Lambda_{i}(1 \leq i \leq r)$ contains at most $2 N / r$ points, and (iv) the number of triangles $\Delta_{i}$ crossed by any line $l$ in the data space is $O(\sqrt{r})=O(\sqrt{B}) . \Delta_{1}, \Delta_{2}, \ldots, \Delta_{r}$ constitute the entries in the root node of the partition tree. The next level is created by constructing balanced simplicial partitions for each $\Lambda_{i}(1 \leq i \leq r)$, and this process is repeated until each partition contains $O(B)$ points, in which case all points in it are stored collectively as a leaf node. Since every point appears in a unique leaf node, the whole tree consumes $O(N / B)$ disk pages. A simplex range query is answered by visiting those nodes whose bounding triangles intersect its search region. Agarwal et al. [AAE+00] show that the query cost is bounded by $O\left((N / B)^{1 / 2+\varepsilon}+K / B\right)$ I/Os, where $\varepsilon$ is an arbitrarily small positive number.

The external priority search tree [ASV99] answers a 3-sided query $q$ optimally. Specifically, given a set $S$ of $N$ 2D points, this tree consumes $O(N / B)$ space, and answers $q$ in $O\left(\log _{B}(N / B)+K / B\right) \mathrm{I} / \mathrm{Os}$, where $K$ is the number of points retrieved. Interestingly, it also optimally solves stabbing queries on $1 \mathrm{D}$ intervals. Specifically, given a set of $N 1 \mathrm{D}$ intervals $\left\{i_{1}, i_{2}, \ldots, i_{N}\right\}$ where $i_{j}=\left[i_{s j}, i_{e j}\right](1 \leq j \leq N)$, a stabbing query specifies a value $q$ and retrieves all intervals $i_{j}(1 \leq j \leq N)$ such that $i_{s j} \leq q \leq i_{e j}$. To solve the problem with the external priority search tree, each interval $i_{j}=\left[i_{s j}, i_{e j}\right]$ is converted to a $2 \mathrm{D}$ point 
$\left(i_{s j}, i_{e j}\right)$; the intervals satisfying $q$ correspond to those points in the 3 -sided rectangle with $\mathrm{x}$-projection $[0, q]$ and y-projection $[q, \infty)$.

\section{Validity Information Retrieval for Range Search}

The result of a RS query $q$ changes (as $q$ or data move) when the boundary of $q$ hits a data point $p$. Thus, we can retrieve, for each edge $e_{i}(1 \leq i \leq 4)$ of $q$, the first point $p_{i}$ that will be hit by $e_{i}$, together with the time $t_{i}$ when this happens. The expiry time $T$ of the current result then equals the smallest $t_{i}(1 \leq i \leq 4)$, and the result change $C$ is due to the point $p_{i}$ with $t_{i}=T$ (i.e., $T$ is the influence time of $p_{i}$ ). Therefore, a validity RS query can be reduced to four dragging queries ${ }^{1}$ each specifying a moving horizontal/vertical line segment (the direction of movement can be arbitrary), and finds the first point it crosses. Consider, for example, Figure $3.1 \mathrm{a}$ with static data points $p_{1}, p_{2}, \ldots, p_{7}$ (similar observations also hold for dynamic objects). The validity RS query $q$ is reduced to dragging queries $e_{1}, e_{2}, e_{3}, e_{4}$ as in Figure $3.1 \mathrm{~b}$, for which the first points hit are $\varnothing, p_{4}$, $\varnothing, p_{6}$, respectively (the result of the validity query is $p_{4}$ since it is crossed earlier than $p_{6}$ ). In the sequel we focus on the vertical dragging query $q$ (the same solutions apply to horizontal queries by symmetry), whose initial position is a vertical line segment at $x=q_{x}$ with $y$-projection $\left[q_{s y}, q_{e y}\right]$ (represented as $q_{x}:\left[q_{s y}, q_{e y}\right]$ ). A query is characterized using two parameters: (i) query length $q_{L}=q_{e y}-q_{s y}$, and (ii) tilting angle $q_{\theta}$ between the query's moving direction and the x-axis, as shown in Figure 3.1c using $e_{4}$ as an example. The subsequent sections solve vertical dragging queries in various problem settings, covering both static data points (Sections 3.1-3.3) and the dynamic ones (Sections 3.4, 3.5).

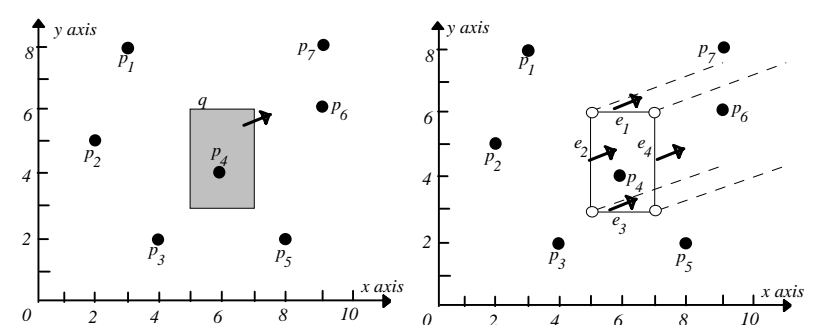

(a) Validity RS query $q$ (b) Four dragging queries

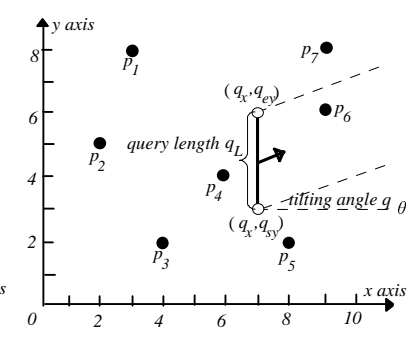

(c) Vertical dragging query

Figure 3.1: Reducing RS validity retrieval to dragging queries

\subsection{Static data and predictable queries}

We first present an optimal solution that consumes linear space $O(N / B)$ and answers a "predictable" dragging query $q$ in $O\left(\log _{B}(N / B)\right)$ I/Os, namely, the query's length and tilting angle (of its movement) are chosen from a constant number of combinations ${ }^{2}$.

\footnotetext{
${ }^{1}$ The term "dragging query" was first used in [C88] for orthogonal line segments with axisparallel movements. Here, we use this term for queries with arbitrary moving directions.

${ }^{2}$ Note that, in practice where query's actual length and moving direction can be measured only discretely, the number of such combinations is indeed constant.
} 
Our solution consists of a set of structures, each one targeting a specific combination. For simplicity, we use queries with length $q_{L}$ moving towards the positive direction of the $\mathrm{x}$-axis; the extension to the other directions is straightforward.

As shown in Figure 3.2a, our goal is to divide the space into disjoint influence areas according to the data points. Specifically, the influence area $A(p)$ of a point $p=\left(p_{x}, p_{y}\right)$ is a 3 -sided rectangle whose projections on the $\mathrm{x}$ - and $\mathrm{y}$ - axes are $\left(-\infty, p_{x}\right)$ and $\left[p_{y}-q_{L} / 2, p_{y}+q_{L} / 2\right]$ respectively, where $q_{L}$ is the targeted query length (e.g., the shaded area in Figure 3.2a represents $A\left(p_{7}\right)$ ). Furthermore, for two data points $p_{i}, p_{j}$ such that $p_{i x}<p_{j x}$ (i.e., $p_{i x}$ is to the "left" of $\left.p_{j x}\right), A\left(p_{i}\right)$ "overwrites" $A\left(p_{j}\right)$ if they overlap (e.g., part of $A\left(p_{6}\right)$ is obstructed by $\left.A\left(p_{7}\right)\right)$. The crucial observation is that, given a dragging query $q=q_{x}:\left[q_{s y}, q_{e y}\right]$, the first point $p$ hit by $q$ is the one whose influence area $A(p)$ covers the query center $\left(q_{x},\left(q_{s y}+q_{e y}\right) / 2\right)$. As an example, the result of the query $q=5:[6,9]$ in Figure 3.2a is $p_{3}$ because its center (the white point) at coordinates $(5,7.5)$ falls into $A\left(p_{3}\right)$. Note that, if such an influence area does not exist, the query result is empty, namely, the dragging query will not hit any point.

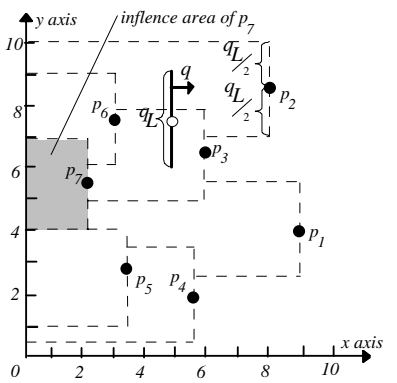

(a) The final areas

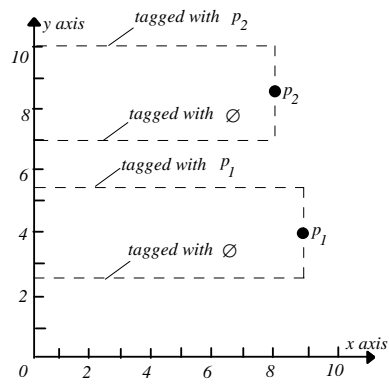

(b) After $p_{1}, p_{2}$ are inserted

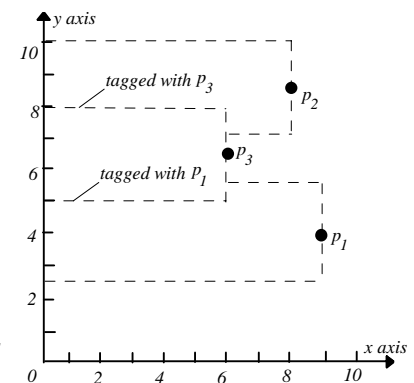

(c) After $p_{3}$ is inserted

Figure 3.2: Computing the influence areas

We use a persistent B-tree to index the influence areas. Specifically, the persistent Btree contains a (logical) $\mathrm{B}$-tree at each $\mathrm{x}$-coordinate $x$, which stores the $\mathrm{y}$-coordinates of the horizontal boundaries of the influence areas spanning $x$. For instance, the B-tree at $x=5$ stores 5 values $\{0.5,3.5,5,8,10\}$, corresponding to the (lower, upper, lower, upper, upper) boundaries of $A\left(p_{4}\right), A\left(p_{4}\right), A\left(p_{3}\right), A\left(p_{3}\right), A\left(p_{2}\right)$, respectively. To construct such a persistent tree, we insert the influence areas of points in descending order of their x-coordinates. In Figure $3.2 \mathrm{~b}, p_{1}$ is the first point processed, after which the persistent tree consists of a single logical B-tree (at the x-coordinate 9 of $p_{1}$ ) containing entries 3.5, 5.5 (i.e., the y-coordinates of the boundaries of $A\left(p_{1}\right)$ ). Entry 5.5 is "tagged" with $p_{1}$ (using constant space), indicating that the region below it belongs to $A\left(p_{1}\right)$, while entry 3.5 is tagged with $\varnothing$, meaning that the region below it is not in any influence area. Similarly, the next point $p_{2}$ inserts two entries 7, 10 (tagged with $\varnothing, p_{2}$ respectively) in the $\mathrm{B}$-tree at $x=8$ (all the $\mathrm{B}$-trees in the $\mathrm{x}$-range $(8,10$ ] have the same content as the one at $x=9)$. Handling the next point $p_{3}$ is more complex since its influence area overlaps $A\left(p_{1}\right)$ and $A\left(p_{2}\right)$. In this case, two entries 5.5 and 7 are removed from the B-tree at $\mathrm{x}=6$, "terminating" the upper and lower boundaries of $A\left(p_{l}\right)$ and $A\left(p_{2}\right)$ respectively. Then, two entries 5, 8 (for the boundaries of $A\left(p_{3}\right)$ ) are inserted into the same tree, after which the tree contains 4 entries $3.5,5,8,10$ tagged with $\varnothing$, $p_{1}, p_{3}, p_{2}$ respectively. The remaining points are inserted in the same way. A dragging 
query $q=q_{x}:\left[q_{s y}, q_{e y}\right]$ is directed to the B-tree at $x=q_{x}$, and finds the smallest entry larger than $\left(q_{s y}+q_{e y}\right) / 2$ (i.e., the y-coordinate of the query center). In Figure 3.2a, for example, the B-tree inspected is at $x=5$, and the entry returned has value 8 . Then, the result (i.e., the point first hit by $q$ ) is the point tagged with the retrieved entry ( $p_{3}$ in the case). The whole processing incurs $O\left(\log _{B}(N / B)\right)$ I/Os.

Theorem 3.1: Given a dataset $S$ of $N$ static 2D points, we can pre-process $S$ into a set of persistent B-trees that occupy totally $O(N / B)$ space and can be constructed in $O\left((N / B) \log _{B}(N / B)\right)$ I/Os, such that a validity RS query $q$ can be answered in $O\left(\log _{B}(N / B)\right)$ I/Os, provided that the query rectangle's size and movement direction are decided from a constant number of combinations.

\subsection{Static data and axis-parallel moving queries}

In this section, we consider (vertical) dragging queries with arbitrary lengths but horizontal movements (i.e., zero tilting angles). It suffices to discuss queries moving towards the positive direction of the $\mathrm{x}$-axis (by symmetry those towards the negative side can be solved in the same way). The first point hit by such a query $q_{x}:\left[q_{s y}, q_{e y}\right]$ is the one with the smallest x-coordinate in the 3 -sided rectangle $\left[q_{x}, \infty\right]:\left[q_{s y}, q_{e y}\right]$. In Figure 3.3, for example, $q=5:[1,8]$ and its 3 -sided rectangle $[5, \infty]:[1,8]$ covers points $p_{l}$, $p_{3}, p_{4}$, among which $p_{4}$ has the smallest x-coordinate and hence, is the result of $q$. Next, we propose two solutions with different tradeoffs between query time and space.

- Using the persistent aggregate tree

We maintain an aggregate $\mathrm{B}$-tree $(\mathrm{aB}$-tree) at every $\mathrm{x}$-coordinate $x$, indexing the $\mathrm{y}$ coordinates of all the points whose $\mathrm{X}$-coordinates are larger than $x$. In addition to the search key, each non-leaf entry of the aB-tree also stores, using $O(1)$ space, the point with the smallest $\mathrm{x}$-coordinate among all the points in its subtree. Figure $3.3 \mathrm{~b}$ demonstrates the aB-tree at $x=5$, where the first root entry $\left\langle p_{4}, 2\right\rangle$, for example, indicates that the smallest $y$-coordinate of all the data points (i.e., $p_{4}, p_{1}$ ) in its subtree is 2 , and $p_{4}$ has the smallest $\mathrm{x}$-coordinate. We store all the aB-trees in a persistent aB-tree, which as shown [TPZ02] $]^{3}$ consumes $O\left((N / B) \log _{B}(N / B)\right)$ space and can be constructed in $O\left((N / B) \log _{B}(N / B)\right) \mathrm{I} / \mathrm{Os}$.

Given a query $q_{x}:\left[q_{s y}, q_{e y}\right]$, the aB-tree at $x=q_{x}$ allows us to answer it in $O\left(\log _{B}(N / B)\right)$ I/Os. To illustrate this, consider the query $(5:[1,8])$ in Figure $3.3 \mathrm{a}$ and the tree in Figure 3.3b. The algorithm starts from the root and descends a non-leaf entry if its "yrange" (enclosing the y-coordinates of points in its subtree) intersects, but is not totally contained in, that of the query ([1,8] in this case). For example, the y-range $[2,6.5]$ of the first root entry in Figure $3.3 \mathrm{~b}$ (where 6.5 is obtained from the second entry) is contained in $[1,8]$, in which case we simply take point $p_{4}$ (stored in this entry) as the candidate result. The subtree of the second entry must be explored because its y-range $[6.5, \infty]$ partially intersects $[1,8]$. In its child node, we examine every data point and update our candidate result accordingly. In this case, $p_{3}$ has larger $\mathrm{x}$ coordinate than $p_{4}$ (our current candidate) while $p_{2}$ does not fall in the 3 -sided rectangle $[5, \infty]:[1,8]$ of the query; hence, no result update is necessary and the algorithm

\footnotetext{
${ }^{3}$ The structure in [TPZ02] is slightly different from the aB-tree in our case, but the complexity analysis still applies.
} 
terminates by returning $p_{4}$. At each level of the aB-tree, the query y-range partially intersects those of at most two entries (notice that the y-ranges of the entries at the same level are disjoint); thus, the algorithm accesses (at most) two complete paths of the tree, i.e., incurring the same complexity as the tree height $O\left(\log _{B}(N / B)\right)$.

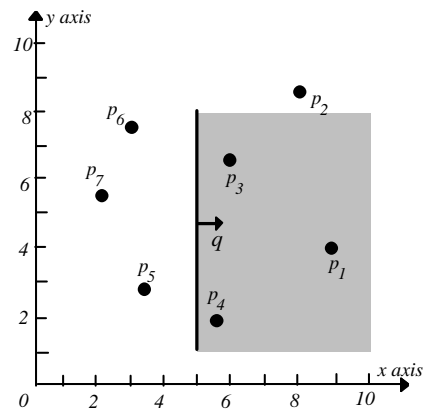

(a) A dragging query

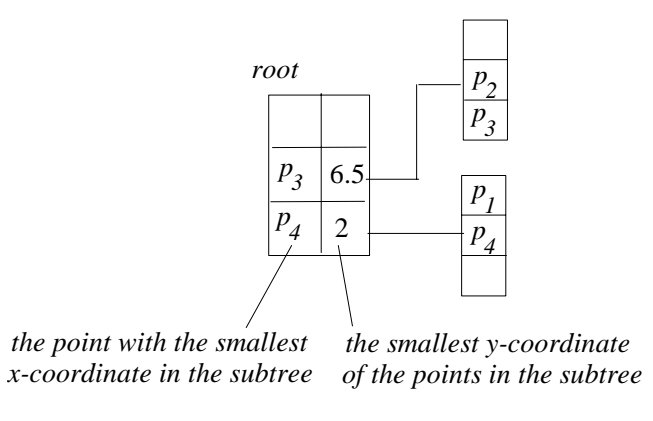

(b) The aggregate B-tree at $x=5$

Figure 3.3: Dragging queries with arbitrary lengths

Theorem 3.2: Given a dataset $S$ of $N$ static 2D points, we can pre-process $S$ into a set of persistent aB-trees that consume totally $O\left((N / B) \log _{B}(N / B)\right)$ space, and can be constructed in $O\left((N / B) \log _{\mathrm{B}}(N / B)\right) \mathrm{I} / \mathrm{Os}$, such that a validity RS query can be answered in $O\left(\log _{B}(N / B)\right)$ time, provided that the moving direction of the query rectangle is axisparallel.

\section{- Using the external range tree}

To decrease the space complexity, we present another solution based on a simplified version of the external range tree. Specifically, as shown in Figure 3.4 (for the data points in Figure 3.3a), the primary structure is a B-tree with fanout $O\left(\log _{B}(N / B)\right)$ built on the $\mathrm{y}$-coordinates of the data points (the height of the tree is thus $\left.O\left(\log _{B}(N / B) / \log \log _{B}(N / B)\right)\right)$. Let $v_{l}, v_{2}, \ldots, v_{r}(r=3$ in Figure 3.4$)$ be the subtrees of an intermediate node $v$ of the tree (e.g., the root of the B-tree). For each branch $v_{i}$ $(1 \leq i \leq r)$, let $\left|v_{i}\right|$ be the number of points it contains, and $p_{i_{1}}, p_{i_{2}}, \ldots, p_{i \mid v_{i}} \mid$ be these points sorted in ascending order of their x-coordinates $p_{i_{1} x}, p_{i_{2} x}, \ldots, p_{i\left|v_{i}\right| x}$. In Figure 3.4, the sorted lists for the root entries $2,4,7.5$ are $\left\{p_{5}, p_{4}\right\},\left\{p_{7}, p_{3}, p_{1}\right\},\left\{p_{6}, p_{2}\right\}$, respectively. We define the interval set of $v_{i}$ as $\left\{\left[-\infty, p_{i_{l} x}\right],\left[p_{i_{1} x}, p_{i_{2} x}\right], \ldots,\left[p_{i\left|v_{i}\right| x}, \infty\right]\right\}$, namely, the intervals produced by the projections of points in $v_{i}$ on the $\mathrm{x}$-axis.

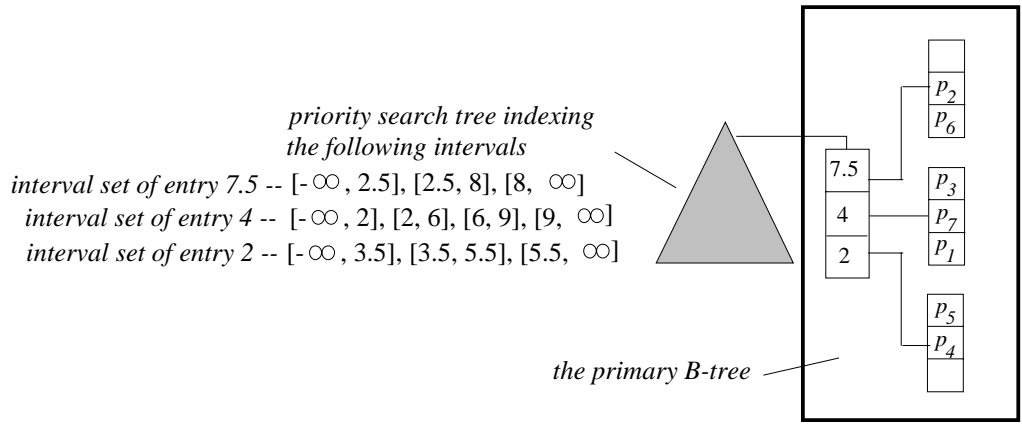

Figure 3.4: A simplified external range tree (for the dataset in Figure 3.3a) 
For example, the interval sets for root entries $2,4,7.5$ in Figure 3.4 are, $\{[-\infty, 3.5]$, $[3.5,5.5],[5.5, \infty]\},\{[-\infty, 2],[2,6][6,9],[9, \infty]\}$ and $\{[-\infty, 2.5],[2.5,8],[8, \infty]\}$. The intervals in the interval sets of all $v_{i}$ are indexed using a priority search tree associated with $v$. Note that, for the non-leaf nodes at the same level of the B-tree, their priority search trees index disjoint sets of intervals, the total number of which is $O(N)$. Given that the height of the primary B-tree is $O\left(\log _{B}(N / B) / \log _{\log }(N / B)\right)$, the total size of the priority search trees (at all B-tree levels) is $O\left((N / B) \log _{B}(N / B) / \log \log _{B}(N / B)\right.$ ), which dominates the B-tree size $O(N / B)$ and constitutes the overall space complexity. This simplified external range tree can be constructed with $O\left((N / B) \log _{B}(N / B)\right) \mathrm{I} / \mathrm{Os}$, same as the complete external range tree [ASV99].

A right-moving dragging query $q=q_{x}:\left[q_{s y}, q_{e y}\right]$ is answered as follows. At the root of the B-tree, we search its associated priority search tree for all the 1D intervals containing value $q_{x}$, which is a stabbing query as reviewed in Section 2. Given the query $q=5:[1,8]$ (Figure 3.3a), for example, this search in the tree of Figure 3.4 returns intervals $[3.5,5.5],[2,6],[2.5,8]$ (containing $q_{x}=5$ ). It is important to note that, each of these intervals comes from the interval sets of distinct subtrees ([3.5,5.5], [2,6], $[2.5,8]$ are from root entries $2,4,7.5$, respectively). Now, let us consider the data points corresponding to the second numbers of these intervals, namely, $p_{4}, p_{3}$, and $p_{2}$ (5.5, for example, is the $\mathrm{x}$-coordinate of $p_{4}$ ). It is safe to conclude that $p_{3}$ is the point first hit by $q$, among all the points in the subtree of entry 4 , whose y-range $[4,7.5)$ is contained in that $[1,8]$ of $q$. Similar conclusions, however, cannot be made for $p_{4}\left(p_{2}\right)$, originating from the subtree of root entry $2(7.5)$, because its y-range $[2,4)([7.5, \infty))$ partially intersects $[1,8]$. In this case, we must visit the child nodes of these entries, where we discover point $p_{4}$ that is hit earlier than $p_{3}$, and becomes the final result.

In general, the above algorithm visits the nodes of the B-tree whose y-ranges partially intersect that of the query. Similar to the case of Figure $3.3 \mathrm{~b}$, the number of such nodes has the same complexity as the tree height, i.e., $O\left(\log _{B}(N / B) / \log \log _{B}(N / B)\right)$. At each node visited, a stabbing query is performed in its associated priority search tree, which returns as many intervals as the node fanout, i.e., $O\left(\log _{B}(N / B)\right)$, incurring $O\left(\log _{B}(N / B)+\log _{B}(N / B) / B\right)=O\left(\log _{B}(N / B)\right)$ I/Os (see the performance of the priority search tree in Section 2). As a result, the total query cost is bounded by $O\left(\log _{B}^{2}(N / B) / \log \log _{B}(N / B)\right)$ I/Os.

Theorem 3.3: Given a dataset $S$ of $N$ static 2D points, we can pre-process $S$ into a set of simplified external search trees that consume $O\left((N / B) \log _{B}(N / B) / \log \log _{B}(N / B)\right)$ space and can be constructed in $O\left((N / B) \log _{B}(N / B)\right)$ I/Os, such that a validity RS query $q$ can be answered in $O\left(\log _{B}^{2}(N / B) / \log \log _{B}(N / B)\right)$ time, provided that the moving direction of the query rectangle is axis-parallel.

\subsection{Static data and arbitrary queries}

This section focuses on dragging queries with arbitrary lengths and tilting angles $q_{\theta}$. Figure 3.5 shows an example query $q_{x}:\left[q_{s y}, q_{e y}\right]$ whose moving direction has slope ${ }^{4}$ $q_{s}=\operatorname{tg}\left(q_{\theta}\right)$. Similar to Figure 3.3a, the goal is to find the point (i.e., $\left.p_{3}\right)$ with the smallest

\footnotetext{
${ }^{4}$ The slope is not defined for tilting angle $q_{\theta}=\pi / 2$ and $3 \pi / 2$, namely, the movement of the query is vertical. This special case is solved in Section 3.2.
} 
$\mathrm{x}$-coordinate among the points in the shaded 3-sided parallelogram. In the sequel, we present two solutions with different characteristics. It is worth mentioning that, these solutions also apply to the problems in the previous two sections, which are special instances of the queries discussed here.

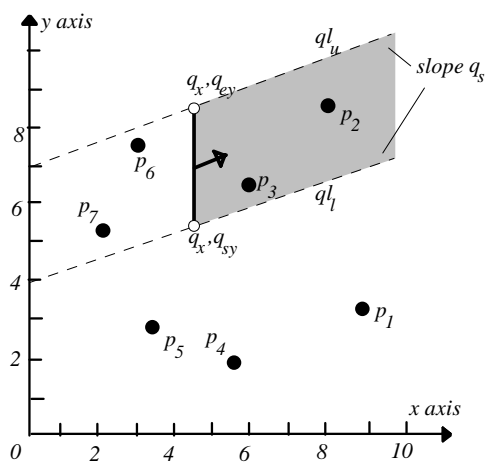

(a) Dragging query and 3-sided parallelogram

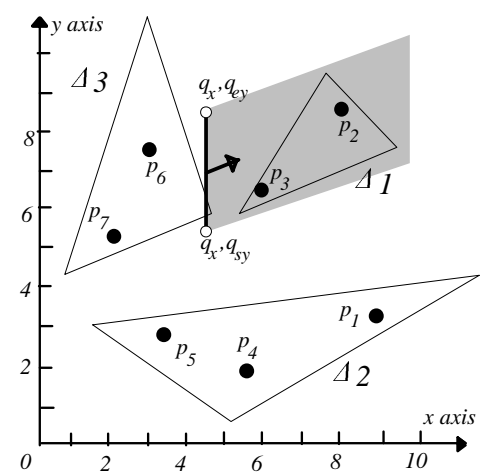

(b) The simplicial partitions

Figure 3.5: Handling dragging queries with non-axis-parallel movement

\section{- Using the partition tree}

As mentioned in Section 2, given a set of $N$ data points, the partition tree answers a simplex query in $O\left((N / B)^{1 / 2+\varepsilon}+K / B\right)$ I/Os (where $\varepsilon$ is an arbitrary positive number, and $K$ is the number of points retrieved). Note that, the parallelogram produced by the dragging query (Figure 3.5a) is indeed a "simplex" shape, i.e., the intersection of three half planes. Applying the partition tree in this case, however, requires solving the following problem: since the goal is to find only one point in the parallelogram, we should avoid paying the extra cost $K / B$ of reporting the other points.

We solve this problem with a slight modification to the partition tree. In each nonleaf node $\Lambda$ of the tree, we store, using $O(1)$ space for each child $\Lambda_{i}$ (which, as described in Section 2, corresponds to a triangular simplicial partition $\Delta_{i}$ ), the point with the smallest $\mathrm{x}$-coordinate in its subtree. Figure $3.5 \mathrm{~b}$ shows an example where the data points are divided into three simplicial partitions $\Delta_{1}, \Delta_{2}, \Delta_{3}$, whose extents are stored in the root of the tree. The points stored for these partitions are $p_{7}, p_{3}, p_{5}$, respectively. This modification does not affect the space and building time complexities of the tree, which, however, can now solve the dragging query in $O\left((N / B)^{1 / 2+\varepsilon}\right) \mathrm{I} / \mathrm{Os}$. Specifically, the search starts from the root, and visits the subtrees whose simplicial partitions partially intersect the 3-sided parallelogram decided by the query. In Figure 3.5b, for example, $\Delta_{l}$ is not accessed because it lies completely in the parallelogram, in which case the data point $p_{7}$ with the smallest $\mathrm{x}$-coordinate is obtained directly from the corresponding root entry. $\Delta_{2}$, on the other hand, must be visited because it partially intersects the shaded region (even though it does not contain any data point inside the parallelogram). Following an analysis similar to that in [AAE+00], we can derive the following theorem.

Theorem 3.4: Given a dataset $S$ consisting of $N$ 2D points, we can pre-process $S$ into a modified partition tree that consumes $O(N / B)$ space such that a validity RS query can be answered in $O\left((N / B)^{1 / 2+\varepsilon}\right) \mathrm{I} / \mathrm{Os}$, for arbitrarily small $\varepsilon>0$. The partition tree can be constructed in $O\left((N / B) \log _{B}(N / B)\right)$ expected I/Os. 


\section{- Using the dual transformation}

We present another solution that reduces general dragging queries (with arbitrary tilting angles) to those with axis-parallel movements, using the dual transformation which converts (i) a point $\left(p_{x}, p_{y}\right)$ in the original space to a line $y=p_{x} \cdot x-p_{y}$ in the dual space, and (ii) a line $y=a \cdot x-b$ to a dual point $(a, b)$. Figure 3.6a illustrates the dual data lines for the points in Figure 3.5a. Given a dragging query $q$, let $q l_{l}$ and $q l_{u}$ be the lines representing the movements of the query segment's upper and lower end-points, respectively (see Figure 3.5a). Since they have the same slope $q_{s}$, their dual points $q p_{l}$ and $q p_{u}$ have the same x-coordinate $q_{s}$. The vertical line segment (we also refer to it as the query segment) in the dual space $q_{s}:\left[q p_{l y}, q p_{u y}\right]$ connecting $q p_{l}$ and $q p_{u}$ has the following important property: it intersects a dual data line (in Figure 3.6a, the intersected lines are $\left.l_{2}, l_{3}, l_{6}, l_{7}\right)$ if and only if the corresponding data point (i.e., $p_{2}, p_{3}, p_{6}, p_{7}$ ) lies between $q l_{l}$ and $q l_{u}$ in the original space.

As shown in Figure 3.5a, the result (i.e., $p_{3}$ ) of the original dragging query $q_{x}:\left[q_{s y}, q_{e y}\right]$ is the point with the smallest x-coordinate among those (i) that lie between lines $q l_{l}$ and $q l_{u}$, and (ii) whose $\mathrm{x}$-coordinates are larger than $q_{x}$. In the dual space, this is equivalent to finding the dual data line with the smallest slope among those (i) that intersect the vertical segment $q_{s}:\left[q p_{l v}, q p_{u v}\right]$, and (ii) whose slopes are larger than $q_{x}$. For this purpose, we perform yet another transformation, which converts a dual data line to a point in the slope-rank space. Specifically, the ranks of dual lines are defined according to their topological ordering at certain $\mathrm{x}$-coordinate (lines may have different ranks at different x-coordinates). In Figure 3.6a, for example, the rank of $l_{2}$ (with slope 8) at $\mathrm{x}=q_{s}$ is 1 , because its intersection with $\mathrm{x}=q_{s}$ is the lowest among the intersection points of all the lines; thus, it is mapped to $(8,1)$ in the slope-rank space. Following the same idea, Figure 3.6b shows the converted points in the slope-rank space of all the lines (with respect to $\mathrm{x}=q_{s}$ ) in Figure 3.6a.

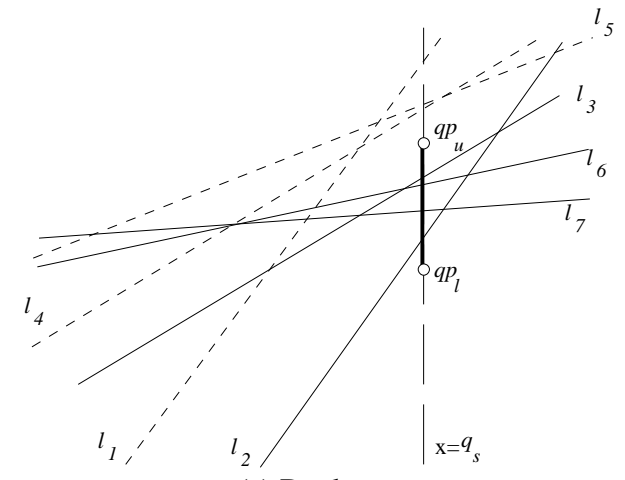

(a) Dual space

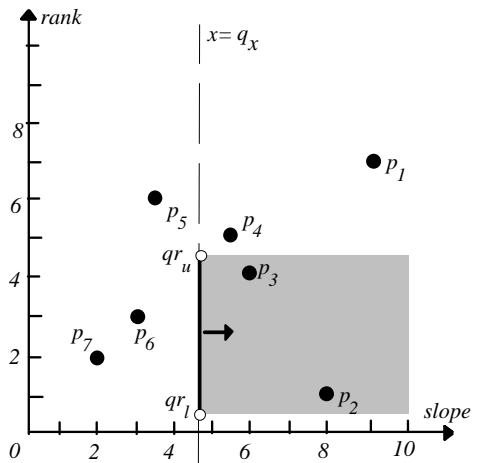

(b) Slope-rank space

Figure 3.6: Using two transformations to solve queries with non-axis-parallel movement

Accordingly, the query segment $q_{s}:\left[q p_{l y}, q p_{u y}\right]$ in the dual space is converted into an other vertical line segment $q_{x}:\left[q r_{l}, q r_{u}\right]$ in the slope-rank space, where $q_{x}$ is the $\mathrm{x}$ coordinate of the original dragging query (in Figure 3.5a, $q_{x}=3.5$ ), and $q r_{l}\left(q r_{u}\right)$ is the rank of point $q p_{l}\left(q p_{u}\right)$ at $\mathrm{x}=q_{s}$ in the dual space. In Figure 3.6, $q r_{l}$ is set to 0.5, indicating that $q p_{l}$ is below all lines at $\mathrm{x}=q_{s}$; similarly $q r_{u}$ is assigned to 4.5 , meaning that $q p_{u}$ is between $l_{4}$ and $l_{3}$ at this X-coordinate. Note that, the values of $q r_{l}$ and $q r_{u}$ are not 
unique; for example, $q r_{l}$ can be any number between 4 (the rank of $l_{4}$ ) and 5 (the rank of $l_{3}$ ), while $q r_{u}$ can be any number below 1 (the rank of $l_{2}$ ). The merit of this transformation is that, now it remains to find, in the slope-rank space, the point first hit by the right-moving vertical dragging query $q_{s}:\left[q r_{l}, q r_{u}\right]$ (i.e., $p_{3}$, which as in Figure $3.5 \mathrm{a}$ is the correct answer to the original dragging query). This problem was solved in Section 3.2; we use the solution based on the simplified external range tree (Figure 3.4).

We have shown that a dragging query whose moving direction has specific slope $q_{s}$, can be solved using an external range tree at $\mathrm{x}=q_{s}$ in the dual space. In order to support all slopes, we must maintain such a tree at every $\mathrm{x}$-coordinate. Fortunately, since we only care about the topological ordering of the dual lines, a new tree is necessary only when the ranks of two lines change. Motivated by this, we adopt the persistent version of the (simplified) external range tree, where a new tree is created at the $\mathrm{x}$ coordinates where two dual lines cross each other. Towards this, the first step is to obtain the intersection points of all pairs of dual lines, using an I/O optimal algorithm for line arrangement computation [GTVV93]. Then the second step constructs the persistent tree in the ascending order of the intersections' $\mathrm{x}$-coordinates. Since there are totally $O\left(N^{2}\right)$ intersections, the space occupied by the persistent tree is $O\left(\left(N^{2} / B\right) \log _{B}(N / B) / \log \log _{B}(N / B)\right)$ and its construction incurs $O\left(\left(N^{2} / B\right) \log _{B}(N / B)\right)$ I/Os.

Further, in order to efficiently assign ranks to the end points of the query segment in the dual space (see Figure 3.6a), we maintain another persistent B-tree where each logical B-tree indexes the ranks of the lines at each $\mathrm{x}$-coordinate in the dual space. This tree consumes $O\left(N^{2} / B\right)$ space, and can be built in $O\left(\left(N^{2} / B\right) \log _{B}(N / B)\right)$ I/Os. The query ranks can be assigned in $O\left(\log _{B}(N / B)\right) \mathrm{I} / \mathrm{Os}$, by searching the B-tree at $\mathrm{x}=q_{s}$ (i.e., the query slope). The total query cost, however, is dominated by that of searching the external range tree, which, as shown in Theorem 3.3, has complexity $O\left(\log _{B}^{2}(N / B) / \log \log _{B}(N / B)\right)$.

Theorem 3.5: Given a dataset $S$ of $N$ static 2D points, we can pre-process $S$ into a set of index structures that consume $O\left(\left(N^{2} / B\right) \log _{B}(N / B) / \log _{\log }(N / B)\right)$ space and can be constructed in $O\left(\left(N^{2} / B\right) \log _{B}(N / B)\right)$ expected I/Os, such that a validity RS query $q$ (with arbitrary window size and moving direction) can be answered in $O\left(\log _{B}^{2}(N / B) / \log \log _{B}(N / B)\right)$ time.

\subsection{Dynamic data and static queries}

Having solved dragging queries on static objects, in this section, we discuss dynamic data points assuming, however, static queries. As before, the goal is to find the first point that crosses the (static) query segment $q=q_{x}:\left[q_{s y}, q_{e y}\right]$. For this purpose, we separate points moving towards the positive/negative direction of the $\mathrm{x}$-axis, and process them independently. Due to the symmetry, it suffices to elaborate our solution for right-moving points. Figure 3.7 a shows an example with 5 points $p_{1}, p_{2}, \ldots, p_{5}$ whose trajectories are represented as rays $l_{1}, l_{2}, \ldots, l_{5}$. For simplicity, assume that all points move at the same speed. Consider two dragging queries $q_{1}, q_{2}$ in Figure 3.7 a, both of which intersect rays $l_{2}$ and $l_{3}$, but in different order. Specifically, for $q_{1}$, the first point hit is $p_{3}$, while for $q_{2}$ the first point is $p_{2}$. Such ordering determines the corresponding query result, and can be described using the concept of "arrival time". Specifically, for each data point $p$ with $\mathrm{x}$-coordinate $p_{x}$, its arrival time $a t_{p}(x)$ for any $x \geq p_{x}$ is the future 
timestamp such that $p_{x}=x$. Then, a dragging query $q=q_{x}:\left[q_{s y}, q_{e y}\right]$ is reduced to finding the point $p$ with the smallest arrival time $a t_{p}\left(q_{x}\right)$, among those whose trajectories intersect the query line segment. As an example, $q_{1}$ intersects $p_{2}$ and $p_{3}$, while $p_{3}$ is the final result since $a t_{p_{3}}\left(q_{1 x}\right)<a t_{p_{2}}\left(q_{1 x}\right)$.

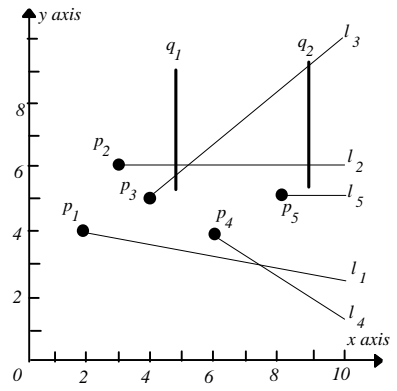

(a) Object trajectories

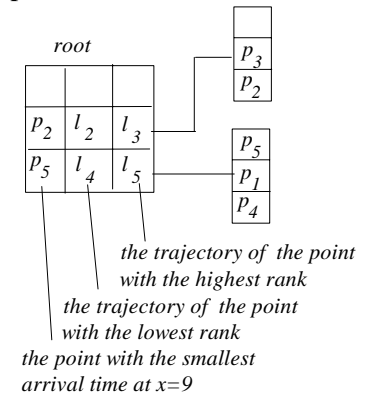

(b) The agg. B-tree at $x=9$

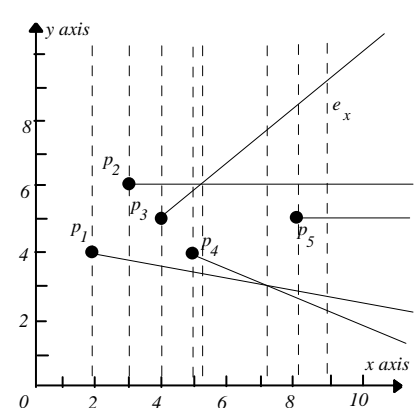

(c) $\mathrm{x}$-coord. for new logical tree

Figure 3.7: Handling dynamic data

Based on this idea, we solve a dragging query using a persistent aggregate tree in logarithmic query cost. Specifically, the persistent tree maintains a logical aggregate B-tree at every $\mathrm{x}$-coordinate $x$ indexing all the data trajectories spanning $x$ (e.g., in Figure 3.7 the logical tree at $\mathrm{x}=9$ stores all rays), which are sorted according to their ranks (i.e., the topological ordering, similar to Figure 3.6) at $x$ (e.g., at $\mathrm{x}=9, l_{4}, l_{3}$ have ranks 1,5 respectively). Furthermore, in each non-leaf entry (of the aggregate tree at $x$ ) we store the ray with the smallest arrival time at $x$ among those in its subtree. In Figure $3.7 \mathrm{~b}$, the first root entry $\left\langle p_{5}, l_{4}, l_{5}\right\rangle$, for example, indicates that at $\mathrm{x}=9$, the point with the lowest (highest) rank in its subtree has trajectory $l_{4}\left(l_{5}\right)$, and the point with the minimal arrival time is $p_{5}$. Given a query $q_{x}:\left[q_{s y}, q_{e y}\right]$, the problem now is reduced to finding the point, in the aggregate tree at $\mathrm{x}=q_{x}$, with the minimal arrival time among those intersecting the query segment which, using the same algorithm given in Section 3.2 , can be solved in $O\left(\log _{B}(N / B)\right) \mathrm{I} / \mathrm{Os}$.

Constructing the persistent aggregate tree deserves further discussion. We deploy a plane-sweep that creates the logical trees from left to right. Specifically, a new logical tree is necessary at the $\mathrm{x}$-coordinates where one of the following events occurs: (i) a new data point appears, (ii) the rays of two points intersect (i.e., the ranks of the rays change), and (iii) the arrival time of two points becomes equal. Figure 3.7c (i.e., the dashed lines) demonstrates all the events for the example of Figure 3.7a. Notice that the x-coordinate $e_{x}$ of the last event (i.e., the right-most dashed line) is due to the fact that the arrival time of $p_{2}$ equals that of $p_{3}$, i.e., $a t_{p_{2}}\left(e_{x}\right)=a t_{p_{3}}\left(e_{x}\right)$. In general, any pair of points $\left(p_{i}, p_{j}\right)$ defines an event (based on their arrival time), if $p_{i \mathrm{x}}>p_{j \mathrm{x}}$ and the speed of $p_{i}$ is larger than that of $p_{j}$. It is easy to see that the number of all events is bounded by $O\left(N^{2}\right)$, and they can be obtained using an algorithm similar to the line arrangement computation given in [GTVV93].

Theorem 3.6: Given a dataset $S$ of $N$ moving $2 \mathrm{D}$ points (with arbitrary velocity), we can pre-process $S$ into a set of aggregate B-trees that consume $O\left(\left(N^{2} / B\right) \log _{B}(N / B)\right)$ space and can be constructed in $O\left(\left(N^{2} / B\right) \log _{B}(N / B)\right)$ expected I/Os, such that a static validity RS query $q$ (with arbitrary window size) is answered in $O\left(\log _{B}(N / B)\right)$. 


\subsection{Dynamic data and dynamic queries}

We discuss the general case where both the data and query are dynamic in the 1D space, and leave the 2D case for future work. Figure 3.8 shows the trajectories $\left(l_{l}, l_{2}\right.$, $\ldots, l_{5}$ ) of 5 points $p_{1}, p_{2}, \ldots, p_{5}$ in the x-time space, and an RS query $q$ with interval $\left[q_{s x}, q_{e x}\right]$ at the current time $t_{c}$ moving as indicated by the arrows. In this case, the corresponding (1D) dragging queries are two "rays" (i.e., $q l_{s}$ and $q l_{e}$ the figure) shooting from the end points of $q$, and each of them finds the point that is first encountered $\left(p_{2}\right.$ and $p_{3}$ respectively). The final answer is the one hit earlier (i.e., $p_{3}$ ).

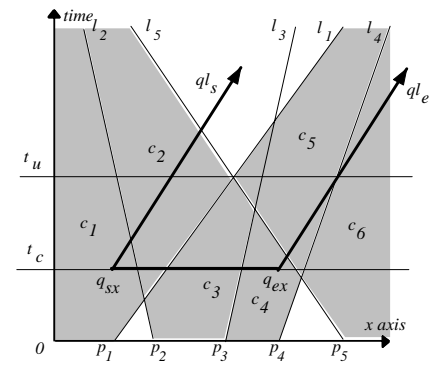

(a) An example

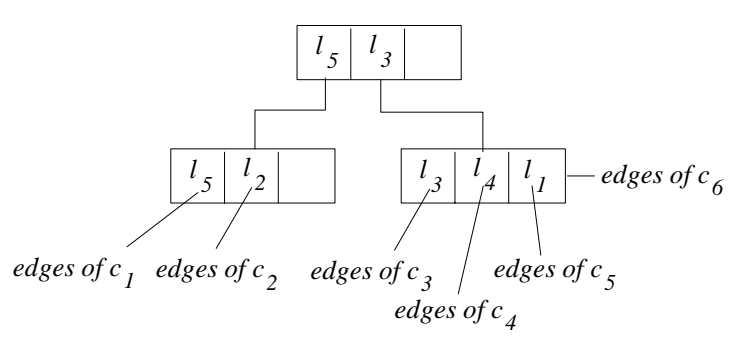

(b) The zone B-tree at $t=t_{c}$

Figure 3.8: Handling moving data and queries

To solve the 1D dragging query, we only need to maintain the zone at the current time $t_{c}$, which consists of all the cells, intersecting line $t=t_{c}$, in the arrangement of the data points' trajectories. Figure 3.8 demonstrates the zone with shaded polygons $c_{1}, c_{2}, \ldots$, $c_{5}$. The zone has complexity $O(N)$ [BKOS97], meaning that the polygons that generate it have $O(N)$ edges (although a single polygon can have $O(N)$ edges in the worst case, the number of such polygons is $O(1)$ ). To answer a dragging query (e.g., $q l_{s}$ in Figure $3.8)$, we first find the cell $c_{l}$ of the zone that covers the source $\left(q_{s x}, q_{t}=t_{c}\right)$ of the query ray, which can be achieved in $O\left(\log _{B}(N / B)\right)$ I/Os, by searching, with value $q_{s x}$, a B-tree (called the zone $B$-tree in the sequel) that indexes the topological ordering of the data trajectories at $t=t_{c}$. In Figure 3.8b, for example, the zone B-tree stores lines $l_{2}, l_{1}, l_{3}, l_{5}$, $l_{4}$, whose ranks at time $t_{c}$ are in this order. Each trajectory is associated with a pointer to the cell (of the zone) to its left. In this example, the cell $c_{l}$ containing $\left(q_{s x}, q_{t}\right)$ is identified through the pointer stored in $l_{2}$. Then, a second search is performed to identify the (unique) edge in $c_{l}$ that is hit by $q l_{s}$. Since $c_{l}$ contains $O(N)$ edges, this can be accomplished in $O\left(\log _{B}(N / B)\right)$ I/Os through, for example, a binary search. Thus, the total query time is $O\left(\log _{B}(N / B)\right) \mathrm{I} / \mathrm{Os}$.

The zone at the current time may become useless as the time progresses. Particularly, the zone changes at discrete timestamps (i.e., events) when the trajectories of two data points cross each other. Following the kinetic approach [BGH97], we manage these events in an event queue, and dynamically maintain the zone at the next event removed from the queue. In Figure 3.8, the next event is at timestamp $t_{u}$, at which time lines $l_{5}$ and $l_{l}$ change their ranks (i.e., ordering). To reflect this, we perform two deletions from the zone B-tree, corresponding to the old ranks of $l_{5}$ and $l_{l}$, and then two insertions for their new ranks. Furthermore, we also need to maintain the cells in the zone, specifically as shown in the figure, removing cell $c_{3}$ that is no longer in the zone, and inserting a new one which is enclosed only by $l_{5}$ and $l_{l}$. Finally, we 
must insert two new events in the event queue, which correspond to the (future) intersection time of $l_{2}$ and $l_{5}, l_{1}$ and $l_{3}$, respectively. In general, a new event is created as a pair of lines become adjacent in the zone B-tree for the first time. Using an external priority queue [A94] as the event queue, handling an event incurs totally $\log _{B}(N / B)$ I/Os.

Theorem 3.7: Given a dataset $S$ of $N$ moving 1D points, we can pre-process $S$ into an index structure that consumes $O(N / B)$ space and can be constructed in $O\left((N / B) \log _{B}(N / B)\right)$ expected I/Os, such that a moving validity RS query $q$ (with arbitrary window sizes and velocities) can be answered in $O\left(\log _{B}(N / B)\right) \mathrm{I} / \mathrm{Os}$. This structure can be updated in $\log _{B}(N / B) \mathrm{I} /$ Os for each kinetic event.

\section{Validity Information Retrieval for Nearest Neighbor}

If the data points are static, validity information retrieval is simple using the Voronoi diagram defined by the objects. Specifically, given a moving query point $q$, we first find the Voronoi cell that contains $q$ (i.e., a point location problem), and then identify the edge of the cell hit by the movement of $q$ (discussed in Section 3.5). The point location problem in the secondary memory can be solved in $O\left(\log _{B}(N / B)\right)$ I/Os, using a persistent B-tree, which (given that the Voronoi cell has complexity $O(N)$ ) consumes $O(N / B)$ space, and can be constructed in $O\left((N / B) \log _{B}(N / B)\right)$ I/Os [ADT03].

Theorem 4.1: Given a dataset $S$ of $N$ static 2D points and its Voronoi diagram, we can pre-process $S$ into an index structure that consumes $O(N / B)$ space and can be constructed in $O\left((N / B) \log _{B}(N / \mathrm{B})\right)$ expected I/Os, such that a moving validity NN query $q$ (with arbitrary moving direction) can be answered in $O\left(\log _{B}(N / B)\right)$ I/Os.

Next, we consider the problem with moving objects in the $1 \mathrm{D}$ space (the $2 \mathrm{D}$ problem is left for future work). Figure 4.1 a shows the trajectories $l_{1}, l_{2}, l_{3}, l_{4}, l_{5}$ of 5 points and a moving query $q$, whose current nearest neighbor is $p_{3}$. In order to find the point that will become the next nearest neighbor of $q$, we mirror $l_{3}$ to $l_{3}$ (i.e., the dotted line) such that the horizontal distance between $l_{3}{ }^{\prime}, q$ is the same as that between $l_{3}, q$ for any future time $t$. In this example, the first points crossed by $p_{3}$ and $p_{3}{ }^{\prime}$ (after the query time) are $p_{1}$ and $p_{5}$ respectively, among which $p_{5}$ is the next NN of $q$. Figure $4.1 \mathrm{~b}$ shows another example, where the trajectories of $q$ and its current $\mathrm{NN} p_{3}$ do not intersect. We mirror $l_{3}$ to $l_{3}$ ' in the same way, and the first point hit by these two lines is $p_{1}$, the next $\mathrm{NN}$ of $q$. The following lemma states that the next $\mathrm{NN}$ of $q$ always come from the first points hit by its current $\mathrm{NN}$ and its mirror.

Lemma 4.1: Given a set $S$ of 1D moving points, let $q$ be the query point whose nearest neighbor is $\rho \in S$. Let $\rho^{\prime}$ be the mirror of $\rho$ with respect to $q$, such that $\forall t>0$, $\operatorname{dist}(\rho(t), q(t))=\operatorname{dist}\left(\rho^{\prime}(t), q(t)\right)$, where $x(\mathrm{t})$ denotes the position of point $x$ at time $t$. Assume that $p_{1}, p_{2}$ are the first points in $S$ that are crossed by $\rho$ and $\rho^{\prime}$ respectively, and let $t_{1}, t_{2}$ be the time when these "crossings" occur. Then the next nearest neighbor of $q$ is $p_{1}$ if $t_{1}<t_{2}$, or $p_{2}$ otherwise.

This lemma permits answering a validity NN query using logarithmic time in the same way as we solved the (1D) dragging query on dynamic data. Specifically, we maintain the zone B-tree as in Figure $3.8 \mathrm{~b}$ so that, given a query point $q$ at the current time, we can efficiently find the cell (in the zone of the current time) that contains $q$ 
(the shaded polygons in Figures 4.1a, 4.1b). Then, its current NN is decided from its neighboring points in the zone B-tree (in both examples of Figure 4.1, $p_{2}, p_{3}$ are the neighboring points, among which $p_{3}$ is the $\mathrm{NN}$ ). Then, deciding the first points hit by the NN and its mirror involves inspecting at most two cells, e.g., two (one) cells in Figure 4.1a (4.1b) (note that the cell containing $q$ is always visited). The zone B-tree and the cells of the zone at the current time can be updated using the kinetic approach as discussed in Section 3.5, with the same query time and space complexities.

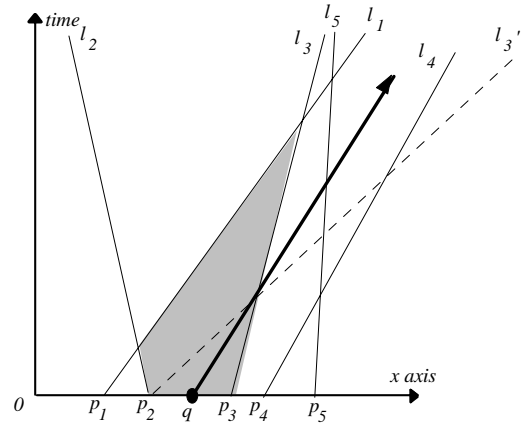

(a) $q$ intersects its $\mathrm{NN}$

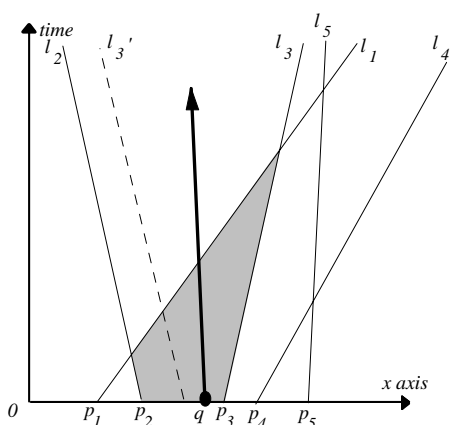

(b) $q$ does not intersect its NN

Figure 4.1: Validity NN query

Theorem 4.2: Given a dataset $S$ of $N$ moving 1D points, we can pre-process $S$ into an index structure that consumes $O(N / B)$ space and can be constructed in $O\left((N / \mathrm{B}) \log _{B}(N / \mathrm{B})\right) \mathrm{I} / \mathrm{Os}$, such that a moving validity RS query $q$ (with arbitrary moving direction) can be answered in $O\left(\log _{B}(N / B)\right)$ I/Os. This structure can be updated in $\log _{B}(N / B) \mathrm{I} /$ Os for each kinetic event.

\section{Summary and Open Problems}

Validity information retrieval aims at accompanying with expiry information the result of a traditional spatial query in a dynamic environment. Therefore, a validity query is usually executed together with the corresponding spatial query and the overall complexity is dominated by the more expensive of these two queries. Table 6.1 summarizes the results in this paper and illustrates the best performance for the corresponding traditional queries. Each cell includes the space complexity, followed by the query time. Using this table, we identify the following open problems:

- For validity RS queries on static data, is there a method which consumes linear space and answers a query in $O\left((N / B)^{1 / 2}\right)$ I/Os? This would eliminate the extra $O\left((N / B)^{\varepsilon}\right)$ query overhead and make the validity query as expensive as the traditional RS, achieving optimal performance using linear space.

- For validity RS queries on static data, is there a method which consumes space $O\left((N / B) \log _{B}(N / B) / \log \log _{B}(N / B)\right)$ and answers an axis-parallel-moving query in $O\left(\log _{B}(N / B)\right)$ I/Os? Such a method would achieve logarithmic query time using the minimal space.

- For static validity RS queries on dynamic data, devise a method which achieves logarithmic query cost with less space consumption than the current solution. 
- Develop index structures for dynamic validity RS queries on dynamic 2D data with good worst case bounds.

- Design a solution that answers NN, and validity NN queries on dynamic 2D data.

- Determine the lower (space/query cost) bounds for the above problems.

\begin{tabular}{|c|c|c|c|}
\hline query & & traditional queries & validity queries \\
\hline \multirow{5}{*}{$\begin{array}{l}\text { range search } \\
\text { on static data }\end{array}$} & \multirow{2}{*}{$\begin{array}{l}\text { linear } \\
\text { space }\end{array}$} & \multirow[t]{2}{*}{$O(N / B), O\left((N / B)^{1 / 2}+K / B\right)[\mathrm{KS} 99, \mathrm{GI} 99]$} & $\begin{array}{l}O(N / B), O\left(\log _{B}(N / B)\right) \text { for constant query } \\
\text { size and velocities }\end{array}$ \\
\hline & & & $O(N / B), O\left((N / B)^{1 / 2+\varepsilon}\right)$ \\
\hline & \multirow{3}{*}{$\begin{array}{l}\text { non- } \\
\text { linear } \\
\text { space }\end{array}$} & \multirow{3}{*}{$\begin{array}{l}O\left((N / B) \log _{B}(N / B) / \log \log _{B}(N / B)\right) \\
O\left(\log _{B}(N / B)+K / B\right)[\text { ASV99] }\end{array}$} & $O\left((N / B) \log _{B}(N / B)\right), O\left(\log _{B}(N / B)\right)$ for $\mathrm{APM}^{*}$ \\
\hline & & & $\begin{array}{c}O\left((N / B) \log _{B}(N / B) / \log \log _{B}(N / B)\right), \\
O\left(\log _{B}^{2}(N / B) / \log \log _{B}(N / B)\right) \text { for APM }\end{array}$ \\
\hline & & & $\begin{array}{c}O\left(\left(N^{2} / B\right) \log _{B}(N / B) / \log \log _{B}(N / B)\right) \\
O\left(\log _{B}^{2}(N / B) / \log \log _{B}(N / B)\right)\end{array}$ \\
\hline \multirow{4}{*}{$\begin{array}{c}\text { range search } \\
\text { on dynamic } \\
\text { data }\end{array}$} & $\begin{array}{l}\text { linear } \\
\text { space }\end{array}$ & $O(N / B), O\left((N / B)^{1 / 2}+K / B\right)[$ ASV99] & NA \\
\hline & \multirow{3}{*}{$\begin{array}{l}\text { non- } \\
\text { linear } \\
\text { space }\end{array}$} & \multirow{3}{*}{$\begin{array}{l}O\left((N / B) \log _{B}(N / B) / \log \log _{B}(N / B)\right) \\
O\left(\log _{B}(N / B)+K / B\right)[\text { ASV99] }\end{array}$} & $\begin{array}{c}O\left(\left(N^{2} / B\right) \log _{B}(N / B)\right), O\left(\log _{B}(N / B)\right) \text { for static } \\
\text { queries }\end{array}$ \\
\hline & & & $O(N / B), O\left(\log _{B}(N / B)\right)$ for 1D data \\
\hline & & & NA for $2 \mathrm{D}$ data \\
\hline $\begin{array}{l}\text { NN on static } \\
\text { data }\end{array}$ & $O(N / B$ & $\begin{array}{l}O\left(\log _{B}(N / B)\right) \text { (Voronoi diagram plus } \\
\text { point location queries) }\end{array}$ & $O(N / B), O\left(\log _{B}(N / B)\right)$ \\
\hline \multirow{2}{*}{$\begin{array}{l}\mathrm{NN} \text { on dy- } \\
\text { namic data }\end{array}$} & & $O(N / B), O\left(\log _{B}(N / B)\right)$ for $1 \mathrm{D}$ & $O(N / B), O\left(\log _{B}(N / B)\right)$ for $1 \mathrm{D}$ \\
\hline & & NA for $2 D$ & NA for $2 D$ \\
\hline
\end{tabular}

*APM= axis-parallel-moving queries

Table 6.1: Summary of the results

\section{Acknowledgements}

This work was supported from grants HKUST 6081/01E, HKUST 6197/02E and HKU 7380/02E from Hong Kong RGC.

\section{References}

[A94] Arge, L. The Buffer Tree: A New Technique for Optimal I/O Algorithms. WADS, 1994.

[AAE+00] Agarwal, P., Arge, L., Erickson, J., Franciosa, P., Vitter, J. Efficient Searching with Linear Constraints. Journal of Computer and System Sciences, 61(2): 194216, 2000.

[AAE00] Agarwal, P., Arge, L., Erickson, J. Indexing Moving Points. ACM PODS, 2000.

[AAV01] Agarwal, P., Arge, L., Vahrenhold, J. A Time Responsive Indexing Scheme for Moving Points. Workshop on Algorithms and Data Structures, 2001.

[ADT03] Arge, L., Danner, A., Teh, S. I/O Efficient Point Location Using Persistent BTrees. ALENEX, 2003.

[ASV99] Arge, L., Samoladas, V., Vitter, J. On Two-Dimensional Indexability and Optimal Range Search Index. ACM PODS, 1999. 
[BGH97] Basch, J., Guibas, L., Hershberger, J. Data Structures for Mobile Data. ACM SODA, 1997.

[BGO+96] Becker, B., Gschwind, S., Ohler, T., Seeger, B. Widmayer, P. An Asymptotically Optimal Multiversion B-trees. VLDB Journal, 5(4): 264-275, 1996.

[BJKS02] Benetis, R., Jensen, C., Karciauskas, G., Saltenis, S. Nearest Neighbor and Reverse Nearest Neighbor Queries for Moving Objects. IDEAS, 2002.

[BKOS97] de Berg, M., van Kreveld, M., Overmars, M., Schwarzkopf, O. Computational Geometry. Springer, 1997.

[BKSS90] Beckmann, N., Kriegel, H., Schneider, R., Seeger, B. The R*-tree: An Efficient and Robust Access Method for Points and Rectangles. ACM SIGMOD, 1990.

[C88] Chazelle, B. An algorithm for segment-dragging and its implementation. Algorithmica, 3: 305-221, 1988.

[CGR95] Callahan, P., Goodrich, G., Ramaiyer, K. Topology B-Trees and Their Applications. Workshop on Algorithms and Data Structures, 1995.

[GBE+00] Guting, R., Böhlen, M., Erwig, M., Jensen, C., Lorentzos, N., Schneider, M., Vazirgiannis, M. A Foundation for Representing and Querying Moving Objects. ACM TODS, 25(1): 1-42, 2000.

[GI99] Grossi, R., Italiano, G. Efficient Splitting and Merging Algorithms for Order Decomposable Problems. Information and Computation, 154(1): 1-33, 1999.

[GLM00] Govindarajan, S., Lukovszki, T., Maheshwari, A., Zeh, N. I/O-Efficient WellSeparated Pair Decomposition and Its Applications. Annual European Symposium on Algorithms, 2000.

[GTVV93] Goodrich, M., Tsay, J., Vengroff, D., Vitter, J. External Memory Computational Geometry. IEEE FOCS, 1993.

[HKP97] Hellerstein, J., Koutsoupias, E., Papadimitriou, C. On the Analysis of Indexing Schemes. ACM PODS, 1997.

[IKO87] Icking, C., Klein, R., Ottmann, T. Priority Search Trees in Secondary Memory. GTCCS, 1987.

[KGT99a] Kollios, G., Gunopulos, D., Tsotras, V. On Indexing Mobile Objects. ACM PODS, 1999.

[KGT99b] Kollios, G., Gunopulos, D., Tsotras, V. Nearest Neighbor Queries in Mobile Environment. STDBM, 1999.

[KRVV96] Kanellakis, P., Ramaswamy, S., Vengroff, D., Vitter, J. Indexing for Data Models with Constraints and Classes. Journal of Computer and System Sciences, 52(3): 589-612, 1996.

[KS99] Kanth, K., Singh, A. Optimal Dynamic Range Searching in Non-Replicating Index Structures. ICDT, 1999.

[RS94] Ramaswamy, S., Subramanian, S. Path Caching: A Technique for Optimal External Searching. ACM PODS, 1994.

[SJLL00] Saltenis, S., Jensen, C., Leutenegger, S., Lopez, M. Indexing the Positions of Continuously Moving Objects. ACM SIGMOD, 2000.

[SR01] Song, Z., Roussopoulos, N. K-Nearest Neighbor Search for Moving Query Point. SSTD, 2001.

[SR95] Subramanian, S., Ramaswamy, S. The P-Range Tree: A New Data Structure for Range Searching in Secondary Memory. ACM SODA, 1995.

[TPZ02] Tao, Y., Papadias, D., Zhang, J. Aggregate Processing of Planar Points. EDBT, 2002.

[TP02] Tao, Y., Papadias, D. Time-Parameterized Queries for Spatio-Temporal Databases. ACM SIGMOD, 2002.

[TPS02] Tao, Y., Papadias, D., Shen, Q. Continuous Nearest Neighbor Search. VLDB, 2002. 